\title{
ORIGINAL ARTICLE Health care utilization in persons with spinal cord injury: part 1-outpatient services
}

\author{
A Gemperli ${ }^{1,2}$, E Ronca ${ }^{1,2}$, A Scheel-Sailer ${ }^{3}$, HG Koch $^{4}$, M Brach ${ }^{1,2}$, B Trezzini ${ }^{1,2}$ and SwiSCI Study Group
}

Study design: This was a cross-sectional questionnaire survey.

Objectives: The objective of this study was to identify the care-seeking behavior of persons with spinal cord injury (SCI) with respect to the various health care providers and ascertain circumstances that lead to situations where required care was not received.

Setting: This study was conducted in the entire country of Switzerland.

Methods: Statistical analysis of frequency of annual visits to health care providers by 17 specialties, and description of situations where health care was required but not received, in persons with chronic SCI living in the community.

Results: Main medical contact person was the general practitioner (GP; visited by $88 \%$ during last 12 months). The physiotherapist (visited by $72 \%$ ) was the health care provider with the most visits (average of 30 visits in 12 months). GPs, physiotherapists, urologists and spinal medicine specialists were often contacted in combination, by many participants, often for check-up visits. A situation where care was required but not received was reported by 53 (11\%) of participants, with a substantially higher rate in migrants (29\%). Main problems why care was not received were bladder and bowel problems and main reasons of care not received were regional or temporal unavailability.

Conclusions: Individuals with $\mathrm{SCl}$ are frequent users of medical services. There is no group of medical specialists that covers all needs of persons with $\mathrm{SCl}$, what emphasizes health care provision from a comprehensive perspective including a wide array of services. Instances with care required but not received appeared to be rare and more likely in participants with migration background.

Spinal Cord (2017) 55, 823-827; doi:10.1038/sc.2017.44; published online 2 May 2017

\section{INTRODUCTION}

Persons with a spinal cord injury (SCI) face a lifelong increased risk of secondary health conditions ${ }^{1}$ and physical decline that leads to higher frequency in utilization of health care services as compared with the general population. ${ }^{2,3}$ Moreover, persons with SCI often require a comprehensive array of general, specialized and home-based health care services. ${ }^{4}$ In these services, providers from different specialties are engaged including medical doctors and therapists, ${ }^{5}$ and complementary and alternative medical services are claimed, based on patient preference. ${ }^{6}$ Besides unplanned visits, as a result of secondary acute complications of SCI, planned visits are commonly offered to persons with SCI as annual check-ups. ${ }^{7}$ Identification of the utilization patterns is important for the effective planning of health care infrastructure under recognition of country characteristics. ${ }^{8,9}$

Switzerland provides an interesting example of an universal health care system in a small, densely populated country where access to all levels of general and specialized medical services without gatekeeping is guaranteed for all residents (Swiss Health Insurance Law) at low individual costs per visit and where supply is generally high (4.0 physicians per 1000 residents; $99.8 \%$ of the population reaches a general hospital within $30 \mathrm{~min}$ by car, $94.0 \%$ within $15 \mathrm{~min}) .{ }^{10}$ However, information on the patterns of health care utilization and identification of unmet health care needs in persons with SCI living in the community in Switzerland are largely missing.
The objective of this study was to determine the proportion of services provided by various health care providers in treating individuals with SCI. Specific aims were: (a) the identification of visiting patterns across the various health care providers; and (b) the determination of the frequency of situations where care was not adequately provided, in general and compared across population subgroups. The study will help to identify the role of the various health care services in the continuum of care, their interrelation and how services are utilized from a user perspective.

\section{MATERIALS AND METHODS \\ Participants}

The study sample was derived from the nationwide community survey within the framework of the Swiss Spinal Cord Injury Cohort Study (SwiSCI). ${ }^{11,12}$ The survey was conducted between late 2011 and early 2013 on the basis of registries from the national association for persons with SCI, three specialized SCI-rehabilitation centers and a SCI-specific home-care institution. Eligible were persons residing in Switzerland with traumatic or non-traumatic SCI aged over 16 years at the time of study. Exclusion criteria comprised congenital conditions leading to SCI, neurodegenerative disorders and Guillain-Barré syndrome. Questionnaires were distributed in three consecutive modules, with the questionnaire on health service utilization being the third module and sent to respondents of the first (69\% response rate) and second ( $84 \%$ response rate of participants of first module) module. ${ }^{13}$ The study was approved by the ethics

\footnotetext{
${ }^{1}$ Rehabilitation Services and Care Unit, Swiss Paraplegic Research, Nottwil, Switzerland; ${ }^{2}$ Department of Health Sciences and Health Policy, University of Lucerne, Lucerne, Switzerland; ${ }^{3}$ Swiss Paraplegic Centre, Nottwil, Switzerland and ${ }^{4}$ Swiss Paraplegics Association, Nottwil, Switzerland Members of the SwiSCI Steering Committee are listed before References.

Correspondence: Dr A Gemperli, Rehabilitation Services and Care Unit, Swiss Paraplegic Research, Guido A. Zäch Strasse 4, Nottwil CH-6207, Switzerland. E-mail: armin.gemperli@paraplegie.ch

Received 4 January 2017; revised 22 March 2017; accepted 23 March 2017; published online 2 May 2017
} 
committees appointed to the specialized rehabilitation centers for SCI, care and patient organizations where the contact addresses were collected.

\section{Measures}

Visits to physicians or therapists were assessed by asking study participants about the frequency of visits to any health care provider of 17 different specialties. These 17 specialties were selected based on a consensus in a group of study stakeholders representing the Swiss specialized rehabilitation centers for SCI, SCI patient and care organizations. It includes medical professions at university educational level, who can bill their services directly to the mandatory (social) health insurance (general practitioner (GP), internist, urologist, neurologist, orthopedic (surgeon), respiratory physician, gynecologist, spinal medicine specialist and the chiropractor), therapeutic services, which are covered by mandatory health insurance only if ordered by a medical doctor and conducted by an accredited therapist (psychologist, speech therapist, occupational therapist, physiotherapist and masseur), dental services, which are not billable to the mandatory health insurance (dentist and dental hygienist), and services by healers. The term healer was not further itemized in the questionnaire, and refers to various alternative and complementary health services, which are loosely regulated and not billable to mandatory health insurance. Study participants reported the number of visits during the last 12 months separately for check-up and acute visits.

Care not received was assessed for all aspects of health care provision. Reported were the number of occasions where medical support was required but not received during the last 12 months. There was no distinction made between whether the problem originated on the care provider's or on the client's side, for example, because of being too busy or because of fears or aversions.

\section{Table 1 Demographics and lesion characteristics of the study population}

Study population, $\mathrm{N}=492$

Sociodemographic characteristics
Males— $n(\%)$
Age in years-mean (s.d.)
Living alone- $n(\%)$
Language region— $n(\%)$
German
French
Italian
Nationality, non-Swiss— $n$ (\%)
Years of education—-mean (s.d.)
Paid work— $n(\%)$
Yes
No
No answer
Household income per month in CHFa_-mean (s.d.)
Health insurance type— $n$ (\%)
Mandatory insurance only
Semi-private insurance
Private insurance
Other model/unknown

$350(71.1)$

$55.3(15.1)$

$113(23.4)$

$347(70.5)$

$122(24.8)$

$23(4.7)$

38 (7.9)

$13.4(3.1)$

$113(23.0)$

194 (39.4)

185 (37.6)

4262 (1785.3)

$344(69.9)$

91 (18.5)

32 (6.5)

$25(5.1)$

$\begin{array}{lr}\text { Lesion characteristics } & \\ \text { Tetraplegia-n (\%) } & 150(30.7) \\ \text { Complete lesion- } n(\%) & 192(39.3) \\ \text { Traumatic etiology- } n(\%) & 373(76.6) \\ \text { Age at onset of SCl—mean (s.d.) } & 38.7(18.2)\end{array}$

Abbreviations: CHF, Swiss francs; SCl, spinal cord injury.

The number of missing values was under $3 \%$ for all items or presented in a separate category.

alncome was measured by net equivalent household income per month in Swiss Francs,

including information on disposable income, household size and number of adults and children according to the Organization for Economic Co-operation and Development (OECD) criteria.
Lesion characteristics sampled were age at SCI onset, time since SCI, SCI etiology, para- or tetraplegia, and completeness of lesion. In distinguishing between para- and tetraplegia, and between complete and incomplete lesion, the International Standards for Neurological Classification of Spinal Cord Injury definition ${ }^{14}$ was not adhered as no clinical assessment was performed. Instead, this information was retrieved from self-reports in the questionnaire. In the questionnaire, complete paralysis was defined as no motor or sensory function preserved below lesion level; otherwise the paralysis was considered incomplete.

Demographic sampled were gender, age, years of education, language (German, French or Italian), level of health insurance coverage (mandatory insurance only, semi-private or private insurance), nationality (Swiss vs non-Swiss), financial situation (financial hardship, disposable household income, invalidity pension coverage), job situation (paid work, occupation), home support (general or professional support), limitation in everyday life and whether living alone or not. Missing information on years of education was re-coded by using free-text information on highest educational degree. The income was converted to net equivalent household income per month, based on information on disposable income, household size and number of adults and children according to the Organization for Economic Co-operation and Development (OECD) criteria. $^{15}$

\section{Statistical analysis}

Demographic information and lesion characteristics of study participants were summarized with absolute and relative frequencies for categorical measures and mean and s.d. for continuous measures. Visits to physicians and therapists were displayed in bar charts showing frequencies for every specialty, subdivided into proportions of check-up and acute visits. Separate figures were produced displaying (1) the relative frequency of participants with at least one visit during the last 12 months by specialty and (2) the average number of visits per person by specialty. The average number of visits per person was derived separately for (a) all study participants and (b) the subset of those who have visited the specific provider during the last 12 months.

Similarity in participants' usage patterns pertaining to different health care providers was depicted in a two-dimensional coordinate plot using multidimensional scaling. First, for every participant a similarity measure between all pairs of health care providers was calculated, expressed as the dichotomous indicator of whether both providers have been contacted during the last year or not. Then that similarity measure was transformed into a single measure of mutual relation between pairs of health care providers by use of the binary Lance-Williams nonmetric dissimilarity measure. ${ }^{16}$ The similarity matrix was then mapped onto a two-dimensional coordinate system using multidimensional scaling. ${ }^{17}$ The mapped values were displayed in a bubble plot with bubble size proportional to total number of utilizations. Thus, the closer the proximity of two health care providers on the chart, relative to all others, the higher the joint usage of their services by the study participants.

Information on care not received was summarized by relative frequency of study participants affected. Further, the five determinants with the largest associations with care not received were identified. These were ascertained by the size of their $P$-values from bivariate logistic regression models with the declaration of care not received as outcome and the respective determinant as independent variable. All $P$-values from comparison with 32 suspected determinants were tested for significance using the Benjamini-Hochberg procedure. $^{18}$

All available data were included in a complete case analysis. Statistical analyses were conducted using Stata version 12 for Windows (College Station, TX, USA).

\section{RESULTS}

\section{Demographics and lesion characteristics}

Five hundred seventy-one survey questionnaires were sent out of which 492 (86\%) were returned. Among the responders, $<4 \%$ of the health utilization questions were not answered. Most participants were male ( $71 \%$ vs $29 \%$ female); mean age was 55 (s.d.: 15.1 ) years with mean age at injury 38 (s.d.: 18.2) years (Table 1). A majority lived 
with paraplegia (69\% vs $31 \%$ tetraplegia) and reported having an incomplete lesion ( $61 \%$ vs $39 \%$ complete SCI), with mostly traumatic etiology ( $77 \%$ vs $23 \%$ non-traumatic SCI).

\section{Utilization of health care providers}

The health service provider visited by most study participants (88\%) during the last 12 months was the GP (Figure 1). Often visited, mainly for check-up visits, were also dentists (76\% of participants) and dental hygienists (58\% of participants). Visited by more than half the participants during the last 12 months were the physiotherapist (72\%), the urologist $(67 \%$ of which $73 \%$ were by men and $27 \%$ by women) and the gynecologist (60\% in women). For acute care most often visited was the GP (46\% of participants), followed by the urologist $(21 \%)$, the physiotherapist and the dentist (18\%, both). The relative frequency of acute care visits among all visits was also the highest for GPs. Of all participants that visited a GP, $54 \%$ did so to receive acute care. A similarly high share of acute care visits were realized by the internist (46\%). Predominantly visited for check-up visits were the dental hygienist (93\% of study participants used it for check-up exclusively), followed by the gynecologist (83\%) and the spinal medicine specialist (80\%).

The health care provider most often visited within 12 months was the physiotherapist with an average of 30 (s.d.: 43.8) visits per participant (Figure 2a). An average of five visits per participant was reported for the GP and the masseur. The highest proportion of acute care visits was reported for the healer (38\% of visits were acute care visits), the neurologist (31\%) and the respiratory physician (30\%). The chiropractor was not often visited, but persons who consulted the chiropractor did so on average 40 times per year (Figure 2b). Most frequent visits by a single person per year had the physiotherapist (maximum of 600 visits), the healer (maximum of 200 visits) and the chiropractor (maximum of 198 visits).

Health care providers frequently visited in combination within 12 months were the GP, the physiotherapist, the urologist, the spinal medicine specialist and the dentist and dental hygienist (Figure 3). Low associations with other health care service utilizations were found for speech therapist, respiratory physician, chiropractor, healer, masseur and psychologist.

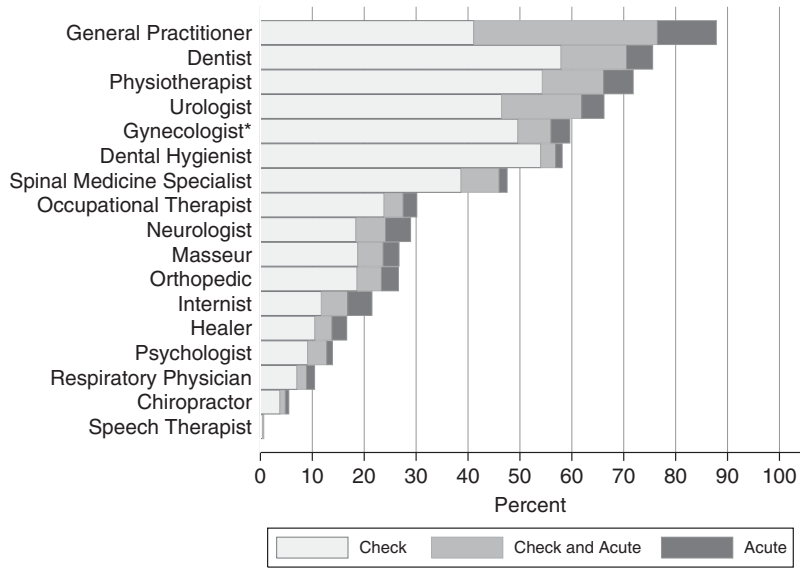

Figure 1 Relative frequency of persons who had at least one medical visit in the last 12 months, by specialty and separated by persons who reported check-up visits only, acute visits only, and those who reported check-up and acute visits. *Visits to the gynecologist for women only.
Failure to receive health care support

Fifty-three participants (11\%) reported at least one occasion where care was not received. A higher frequency was reported in immigrants ( $29 \%$ vs $10 \%$ in Swiss) and in participants with home support (14\% vs $6 \%$ for participants without home support). However, none of these group differences was found to be statistically significant when adjusting for multiple testing. Of the 53 participants who reported care not received, ten did not report the number of occasions and the other 42 participants mentioned a median value of two occasions (interquartile range 1-3) during the last 12 months. Eight participants (19\%) reported five or more occasions. The mean number of occasions in this population was 4 (s.d. 8.3). More issues were reported in participants with SCI caused by a leisure activity (mean increase 6; 95\% confidence interval: $0.2,10.9$ ) and in homemakers (mean increase $7 ; 95 \%$ confidence interval: $0.1,13.1$ ).

The most frequently mentioned health problems for which health care support was required, but not received or sought, were bladder problems (47\%) followed by bowel problems (32\%) and medication needs $(25 \%)$. Although respondents did not often mention wound treatment, respiratory problems and autonomic dysreflexia, they

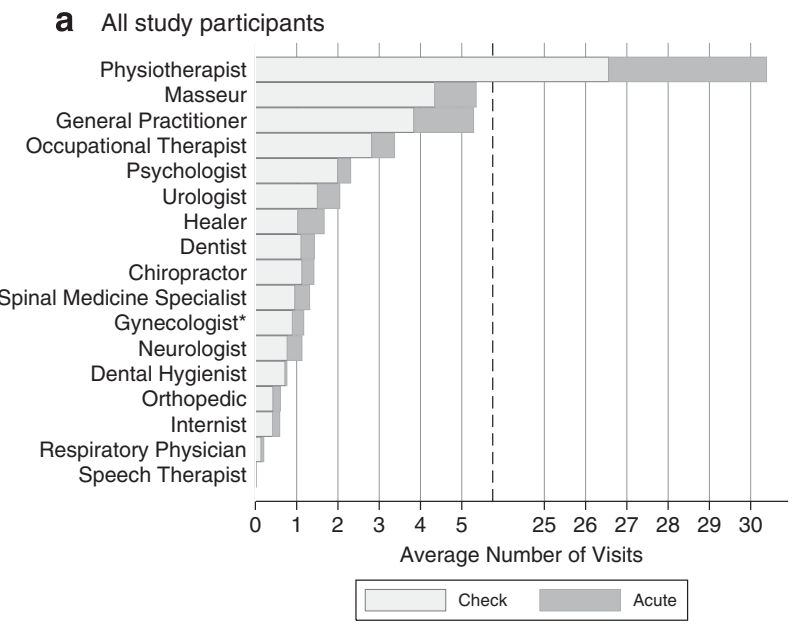

b Study participants with at least one visit to the respective health care provider

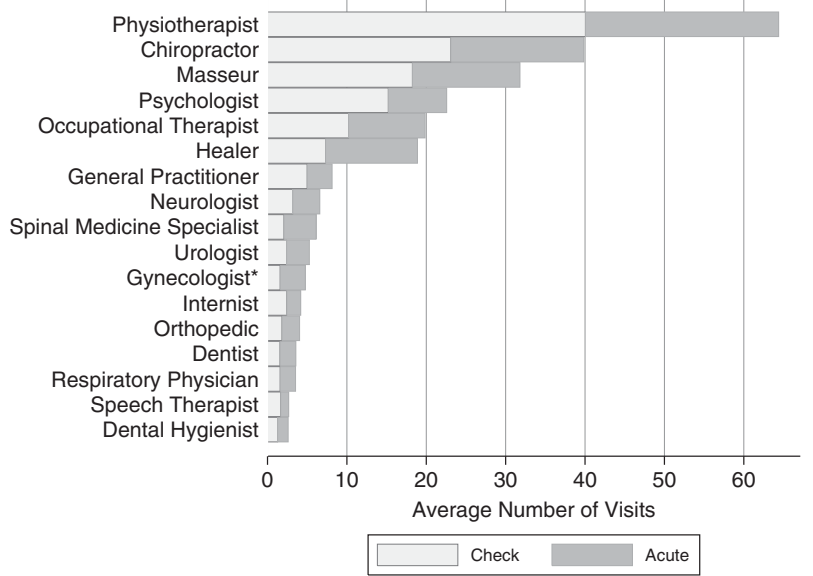

Figure 2 Average number of medical visits per person in the last 12 months, by specialty and separated by check-up visits and acute visits. *Visits to the gynecologist for women only. The average number of annual visits for every participant was determined separately for acute and check-up visits. 


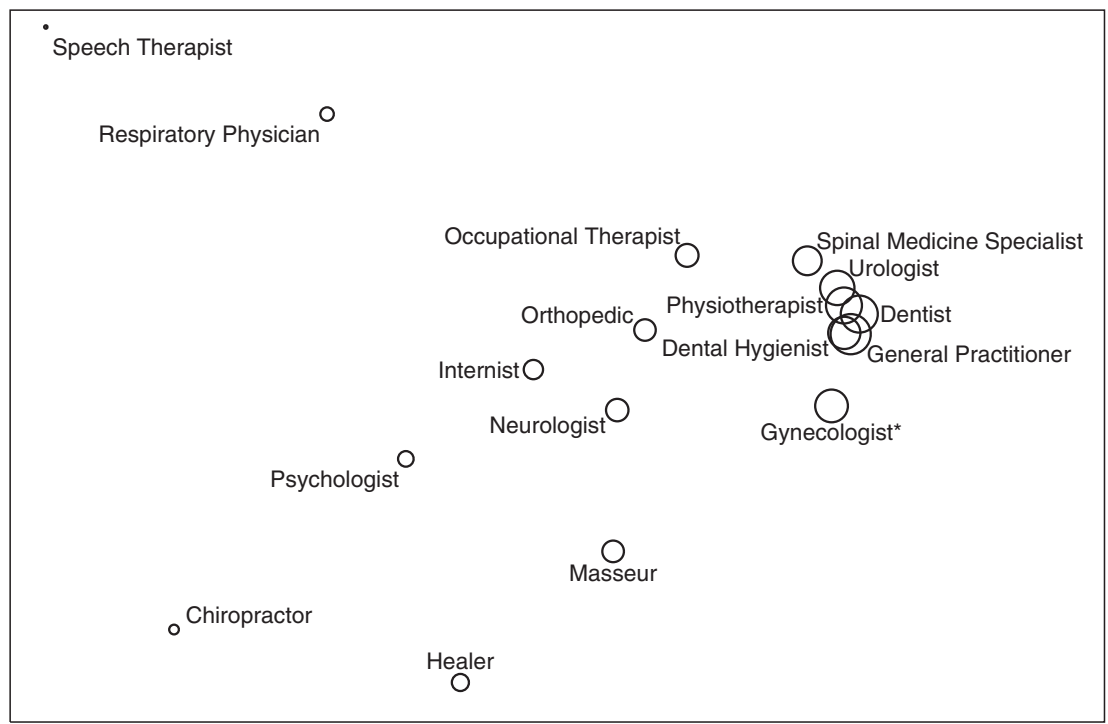

Figure 3 Patterns of similar use of different health care services in the last 12 months. The closer the specialties depicted, the more likely they have jointly been used during the last 12 months. The larger the bubble size, the higher the relative frequency of service use. Mapping produced with multidimensional scaling of similar usage of health care services measured by the Lance-Williams metric. *Visits to the gynecologist for women only.

highlighted many different issues over the entire range of SCI-specific secondary health conditions (42\%).

As main reasons for not receiving health care support, the participants reported unavailability of the services at a specific time $(26 \%)$ or in their region of residence $(23 \%)$. Further reasons were high costs, lack of knowledge on who needs to be contacted (both $15 \%$ ), long waiting time (13\%) or a lack of SCI expertise of the health care provider (11\%).

\section{DISCUSSION}

This study illustrates a well-functioning health system characterized by high utilization in a wide array of services for those in need. Identified as main health care providers for persons with SCI were GPs and physiotherapists. Health care providers with relatively few clients but many visits were chiropractor, masseur and psychologist. Chiropractors are in Switzerland legally recognized like regular doctors (Federal Law on Medical Professions). Patients can freely choose their chiropractor, chiropractors are allowed to bill to the mandatory health insurance and every mandatory insurance plan must cover their services. In Switzerland, visits to the masseur are financially covered under extra insurance coverage only. The $27 \%$ of participants who visited the masseur had on average 32 visits (maximum 104 visits or 2 per week) per year and the $14 \%$ who went to the psychologist 23 (maximum 120 visits). In contrast, service providers, which cover a higher proportion of participants but with fewer visits, were those most often providing regular check-up visits, such as the dentist, the dental hygienist and the gynecologist (all $<5$ visits a year on average). Depending on the Swiss canton, healers are either regulated, tolerated or prohibited, but generally popular (visited by $17 \%$ of participants during the last year). Healers must be paid out-of-pocket. Non-medical psychotherapy and services by psychologists are only covered by the mandatory health insurance by referral of a doctor, but still used by $14 \%$ of study participants. The relatively high utilization of health services not covered by the mandatory health insurance suggests an inadequate coverage of those services for the SCI population and a notable willingness to pay for these services either out-of-pocket or via extra insurance.
Related health care utilization (Figure 3) was demonstrated for primary care providers as one group, SCI specialist services and practical SCI service providers as other groups. Somewhat detached of utilization of these services were the neurologist and psychologist, with the latter attributed to a larger cluster of complementary and alternative services constituted by the chiropractor, the masseur and the healer. These findings suggest that complementary and alternative medical services were utilized in exchange rather than in addition to other services.

A moderate number of participants (11\%) reported situations where care was not received during the last 12 months, but the number of issues mentioned by these persons were many. Participants reporting these problems were identified as being less familiar with the health system (foreign nationality). This finding does not match those of other studies where elevated expectations in persons with high income and education more likely lead to dissatisfaction with health care provision. ${ }^{19}$ The health problems for which care was required but not received were those typical for persons with SCI (e.g. bladder and bowel problems). ${ }^{1}$ Access barriers were reported as main reasons, which reflects other findings of lower accessibility of fee-for-service practices-as established in Switzerland-in comparison with salaried or capitation practices for persons with disability. ${ }^{20}$

The study illustrates the implications and usage patterns in a universal health care system that guarantees affordable access to a wide spectrum of health care services including specialist medicine without gatekeeping and some complementary and alternative medical services. High utilizations were found in all services and the combined and subsidiary usage of many service providers are equally emphasized. A moderate number of situations of unmet health care requirements was identified, linked to barriers in availability of local services, especially for those less familiar with the health care system.

\section{Study limitations}

This cross-sectional study was based on self-reported health care utilization. The validity of the sample has been investigated via a comparison of demographic information and lesion characteristics between non-responders and the study sample. The study sample was 
found highly representative of the invited population. ${ }^{12}$ No conclusion can be drawn regarding the general Swiss SCI population, however, the study setup is designed to systematically include all eligible individuals. ${ }^{11}$ The survey did not apply international standards for sampling lesion characteristics, such as in determining level and completeness of lesion, as no clinical assessment was able to be conducted as part of the home-based questionnaire. This not only produced imprecise information on lesion characteristics, it also prevents comparison with other studies. A further problem with self-reporting is recall bias, especially in high users of health care services when surveying number of utilizations. ${ }^{21,22}$ The higher challenge to recall frequent utilizations let us assume that the study more likely under- than overestimates the number of utilizations. ${ }^{23}$ In the KORA study in a population aged 65 years and older, the self-reported number of physician visits underestimated true utilization by $70 \% .^{22}$

\section{CONCLUSIONS}

The lack of regulation for alternative and complementary medicine in Switzerland leads to a substantial amount of utilization of niche factors such as chiropractors and healers. These services do not interfere much with traditional service providers but build a separate framework for a clientele who is willing to pay for these services either out-of-pocket or via extra insurance. Obstructions in health care access is at a moderate level and concentrated in migrants who may have a limited understanding of the service infrastructure. The study highlights the interplay between a large number of general and specialist services for acute care and opportunities for check-up visits as important constituents of a comprehensive health care system for the benefit of persons with chronic SCI.

\section{DATA ARCHIVING}

There were no data to deposit.

\section{CONFLICT OF INTEREST}

All authors were salaried by organizations financially compensated by the Swiss Paraplegic Foundation.

\section{ACKNOWLEDGEMENTS}

We are grateful to all the participants of the SwiSCI survey for their time and effort spent in responding to the questions. We thank Ingrid Tanner for editing of the manuscript. This study has been financed in the framework of the SwiSCI study, supported by the Swiss Paraplegic Foundation.

\section{SWISCI STUDY GROUP}

The members of the SwiSCI Steering Committee are: Xavier Jordan, Bertrand Léger (Clinique Romande de Réadaptation, Sion); Michael Baumberger, Hans Peter Gmünder (Swiss Paraplegic Center, Nottwil); Armin Curt, Martin Schubert (University Clinic Balgrist, Zürich); Margret Hund-Georgiadis, Kerstin Hug (REHAB Basel, Basel); Hans Georg Koch (Swiss Paraplegic Association, Nottwil); Nadja Münzel (Parahelp, Nottwil); Hardy Landolt (Representative for persons with SCI, Glarus); Mirjam Brach, Gerold Stucki (Swiss Paraplegic Research,
Nottwil); Martin Brinkhof, Christine Thyrian (SwiSCI Study Center at Swiss Paraplegic Research, Nottwil).

1 Jensen MP, Truitt AR, Schomer KG, Yorkston KM, Baylor C, Molton IR. Frequency and age effects of secondary health conditions in individuals with spinal cord injury: a scoping review. Spinal Cord 2013; 51: 882-892.

2 Dryden DM, Saunders LD, Rowe BH, May LA, Yiannakoulias N, Svenson LW et al. Utilization of health services following spinal cord injury: a 6 -year follow-up study. Spinal Cord 2004; 42: 513-525.

3 Krause JS, Cao Y, Bozard JL. Changes in hospitalization, physician visits, and self-reported fitness after spinal cord injury: a cross-sequential analysis of age, years since injury, and age at injury onset. Arch Phys Med Rehabil 2013; 94: 32-37.

4 Beatty PW, Hagglund KJ, Neri MT, Dhont KR, Clark MJ, Hilton SA. Access to health care services among people with chronic or disabling conditions: patterns and predictors. Arch Phys Med Rehabil 2003; 84: 1417-1425.

5 Hamilton R, Driver S, Noorani S, Callender L, Bennett M, Monden K. Utilization and access to healthcare services among community-dwelling people living with spinal cord injury. J Spinal Cord Med (e-pub ahead of print 25 May 2016; doi:10.1080/ 10790268.2016.1184828).

6 Pannek J, Pannek-Rademacher S, Wöllner J. Use of complementary and alternative medicine in persons with spinal cord injury in Switzerland: a survey study. Spinal Cord 2015; 53: 569-572.

7 Spreyermann R, Lüthi $\mathrm{H}$, Michel $\mathrm{F}$, Baumberger ME, Wirz M, Mäder M. Long-term follow-up of patients with spinal cord injury with a new ICF-based tool. Spinal Cord 2011; 49: 230-235.

8 Donnelly C, McColl MA, Charlifue S, Glass C, O'Brien P, Savic G et al. Utilization, access and satisfaction with primary care among people with spinal cord injuries: a comparison of three countries. Spinal Cord 2007; 45: 25-36.

9 New PW, Townson A, Scivoletto G, Post MWM, Eriks-Hoogland I, Gupta A et al. International comparison of the organisation of rehabilitation services and systems of care for patients with spinal cord injury. Spinal Cord 2013; 51: 33-39.

10 Credit Suisse Group. Gesundheitswesen Schweiz 2013: Der Spitalmarkt im Wandel [in German]. Credit Suisse, 2013.

11 Post MWM, Brinkhof MWG, von Elm E, Boldt C, Brach M, Fekete C et al. Design of the Swiss spinal cord injury cohort study. Am J Phys Med Rehabil 2011; 90: S5-16.

12 Brinkhof MWG, Fekete C, Chamberlain JD, Post MWM, Gemperli A, SwiSCl Study Group. Swiss national community survey on functioning after spinal cord injury: protocol, characteristics of participants and determinants of non-response. J Rehabil Med 2016; 48: 120-130.

13 Fekete C, Segerer W, Gemperli A, Brinkhof MWG, SwiSCI Study Group. Participation rates, response bias and response behaviours in the community survey of the Swiss Spinal Cord Injury Cohort Study (SwiSCI). BMC Med Res Methodol 2015; 15: 80.

14 Kirshblum SC, Burns SP, Biering-Sorensen F, Donovan W, Graves DE, Jha A et al. International standards for neurological classification of spinal cord injury (Revised 2011). J Spinal Cord Med 2011; 34: 535-546.

15 Hagenaars AJM, De Vos K, Zaidi MA. Poverty statistics in the late 1980s: research based on micro-data, study carried out for Eurostat 1994.

16 Anderberg MR. Cluster Analysis for Applications. Academic Press, New York and London. 1973.

17 Torgerson WS. Multidimensional scaling: I. Theory and method. Psychometrika 17: 401-419.

18 Benjamini Y, Hochberg Y. Controlling the false discovery rate: a practical and powerful approach to multiple testing. J R Stat Soc Ser B Methodol 1995; 57: 289-300.

19 McColl MA, Jarzynowska A, Shortt SED. Unmet health care needs of people with disabilities: population level evidence. Disabil Soc 2010; 25: 205-218.

20 McColl MAH, Shortt S, Hunter D, Dorland J, Godwin M, Rosser W et al. Access and quality of primary care for people with disabilities: a comparison of practice factors. J Disabil Policy Stud 2010; 21: 131-140.

21 Jordan K, Jinks C, Croft P. Health care utilization: measurement using primary care records and patient recall both showed bias. J Clin Epidemiol 2006; 59: 791-797.

22 Hunger M, Schwarzkopf L, Heier M, Peters A, Holle R, KORA Study Group. Official statistics and claims data records indicate non-response and recall bias within survey-based estimates of health care utilization in the older population. BMC Health Serv Res 2013; 13: 1

23 Icks A, Dittrich A, Brüne M, Kuss O, Hoyer A, Haastert B et al. Agreement found between self-reported and health insurance data on physician visits comparing different recall lengths. J Clin Epidemiol 2016; 82: 167-172. 